\title{
OSPP Face Recognition Using Meta-Heuristic Algorithm
}

\author{
Syed ArshiRahaman \\ ${ }^{1}$ (Computer Science and engineering, BankuraUnnayani Institute of Engineering,India)
}

\begin{abstract}
Face recognition has drawn dramatic attention due to the advancement of pattern recognition technologies. Face recognition systems have reached a level of maturity under certain conditions but still the performance of face recognition algorithms are easily affected by external and internal variations. Thus many well-known algorithms have been proposed to overcome these challenging problems. Here we are trying to use one sample face image of individual for training the whole system which will not only reduce labouring effort for the collection and also reduce cost for storing and processing them. One sample per person face recognition $(O S P P)$ is considered as a challenging problem in face recognition community and lack of samples leads to performance deterioration. Here face recognition is performed by application of the swarm optimization algorithms ${ }^{[12]}$. It was found out that the underlying foraging principle and the swarm optimization can be integrated into evolutionary computational algorithms to provide a better search strategy for finding optimal feature vectors for face recognition. Finally, it is believed that the particle swarm optimization may be useful for the development of face recognition system. A meta-heuristic algorithm PSO is used that makes few or no assumptions about the problem being optimized and can search very large spaces of candidate solutions and also used for classifying purpose.
\end{abstract}

Keywords: One sample per person, particle swarm optimization, meta-heuristic algorithm, face recognition, intra-class variety model

\section{Introduction}

The fundamental task of face recognition technology is to recognize a human face. Here we consider the images (training set) of different subject and then extract the features from them. the next step includes training the system for classification purpose. The same architecture is followed and one sample per person is considered for the training purpose and for classification purpose Meta-heuristic algorithm like PSO is used. Particle Swarm Optimisation is an algorithm capable of optimising a non- linear and multidimensional problem which usually reaches good solutions efficiently while requiring minimal parameterisation. The basic concept of the algorithm is to create a swarm of particles which could move in the space around the problem space searching for their goal, the place which best suits their needs given by a fitness function. OSPP face recognition algorithm is based on PSO. If we consider a neutral image of each subject as a particle, each particle has a specific mode of direction (mode of direction has been discus in proposed model). In each iteration every particle moves towards optimal solution based on the cost function. after a specific amount of iterations, the particle holding best cost function will be the optimal point (solution).

Particle swarm optimisation is chosen to find out the optimum combination of the basis and variety in terms of the minimum L2 distance relative to the query image. The particles are scattered randomly in the search space. As they search for the optimum value, the particles balance the objectives of following the value gradient and random exploration. Over time they begin to congregate in the general area of the optimum value. Finally, the particles converge to the optimum value in the concept. Experiments on the extended Yale face database are provided to show the validity of the proposed algorithm.

\section{OSPP Problems}

\section{Materials and methods}

Accuracy is generally proportional to the number of training samples collected for face recognition. The "one sample per person" (OSPP) problem focuses on identifying a person using only one training sample. The facial recognition from a single sample reduces the labour required to gather training data and enables some applications where only a single sample will be available.

Based on the features used in OSPP, the problem can be classified into three part

- Holistic methods: These methods identify a face using the whole face image as an input. The main challenge faced by these methods is how to address the extremely small sample problem. such as Extensions of principalcomponent analysis (PCA) enriched face images with its projections.

( PC) $^{2}$ A: Two-dimensional PCA ${ }^{[2]}$

- Local methods: These methods use the local facial features for recognition. Care should be taken when deciding how to incorporate global confrontational information into local face model. Such as DCP Use 
directional corner points (DCP) features for recognition Graph matching methods ${ }^{[3-6]}$ Local probabilistic subspace Local probabilistic subspace method Neural network method SOM learning based recognition

Hidden Markov Model HMM method

- Hybrid methods: These methods use both the local and holistic features to recognize a face. These methods have the potential to ołer better performance than individual holistic or local methods, since more comprehensive information could be utilised. Such as Virtual samples + local features Local probabilistic subspace method

\section{Particle Swarm Optimisation}

Particle Swarm Optimization belongs to the field of Swarm Intelligence and Collective Intelligence and is a sub-field of Computational Intelligence. The goal of the algorithm is to have all the particles locate the optima in a multi-dimensional hyper-volume. This is achieved by assigning initially random positions to all particles in the space and small initial random velocities. The algorithm is executed like a simulation, advancing the position of each particle in turn based on its velocity, the best known global position in the problem space and the best position known to a particle. The objective function is sampled after each position update. Over time, through a combination of exploration and exploitation of known good positions in the search space, the particles cluster or converge together around an optima, or several optima.

\section{General Equation of PSO}

If the particles are in .M-dimensional space then the $i^{t h}$ particle can be represented as $X=\left[X_{1}^{i}, X_{2}^{i} \ldots . . X_{M}^{i}\right]$ and the position of the particles $i$ can be represented as $P^{i}=\left[P_{1}^{i}, P_{2}^{i} \ldots . . P_{M}^{i}\right]$. now thePosition of all particle is $P_{g, t}\left(\right.$ Global Best) and the best Position of $i^{t h}$ particle is $P_{g, t}^{i}($ Personal Best $)$. Where $t$ is the iteration no. the rate of Position change of $i^{t h}$ particle is noted as $V^{i}=\left[V_{1}^{i}, V_{2}^{i} \ldots . V_{M}^{i}\right]$

The position change of $i^{\text {th }}$ particle can Modified according to

$$
\begin{gathered}
V_{i, t+1}=w * V_{i, t}+C_{1} * \operatorname{rand}(\quad) *\left(P_{g, t}^{i}-X_{t}^{i}\right)+C_{2} * \operatorname{rand}(\quad) *\left(P_{g, t}-X_{t}^{i}\right) \\
\text { and } \\
X_{t+!}^{i}=X_{t}^{i}+V_{i, t+1}
\end{gathered}
$$

where $C_{1}, C_{2}$ are positive constant. and rand() is a random function.

\section{OSPP Face Recognition Using PSO}

One sample per person face recognition on Particle swarm optimisation was introduced by Yan Zhang and Hua Peng. The Basis plus variety model ${ }^{[11]}$ was also introduced in OSPP-PSO .Neutral images of each subject were considered as particles and the rate of position changes of each particles were initiated with intra class variety. $\mathrm{K}$ no of subjects were taken into consideration and each sample holds $\mathrm{M}$ no of samples and 1 neutral image $X_{1}^{j}, j=1,2,3 \ldots \ldots . k$

Here for subject $j, j=1,2,3 \ldots \ldots . k$, and $r$, th sample $r=1,2,3 \ldots \ldots . M$.to initiate movement rate of each particle (Neutral Image )the intra-class variation was used.

Theintra-class variation was once expressed as

$V=\left[V_{1}, V_{2} V_{3} \ldots \ldots V_{m-1}\right]=\left[\frac{!}{k} \sum_{i=1}^{k}\left(V_{2}^{i}-V_{1}^{i}\right) \ldots \ldots \ldots \ldots ! \frac{!}{k} \sum_{i=1}^{k}\left(V_{r}^{i}-V_{1}^{i}\right) \ldots \frac{!}{k} \sum_{i=1}^{k}\left(V_{M}^{i}-\right.\right.$

$V 1 i \ldots \ldots \ldots \ldots . . . . . . . . . . . .(1)$

\section{Proposed Model}

To initiate rate of position changes of each particle we have used difference of intra-class average face and neutral face of same class instead of intra-class variation.

$$
\begin{gathered}
V_{i}=\left(X_{1}^{i}-\frac{1}{M} \sum_{n=1}^{M} X_{i}^{n}\right)=[\text { Neutral face }(i)-\text { Average face }(i)] \\
i=1,2,3 \ldots \ldots, \text { and } X_{1}^{i} \text { is the neutral image for class } i
\end{gathered}
$$




\section{System flowchart of Proposed Model}

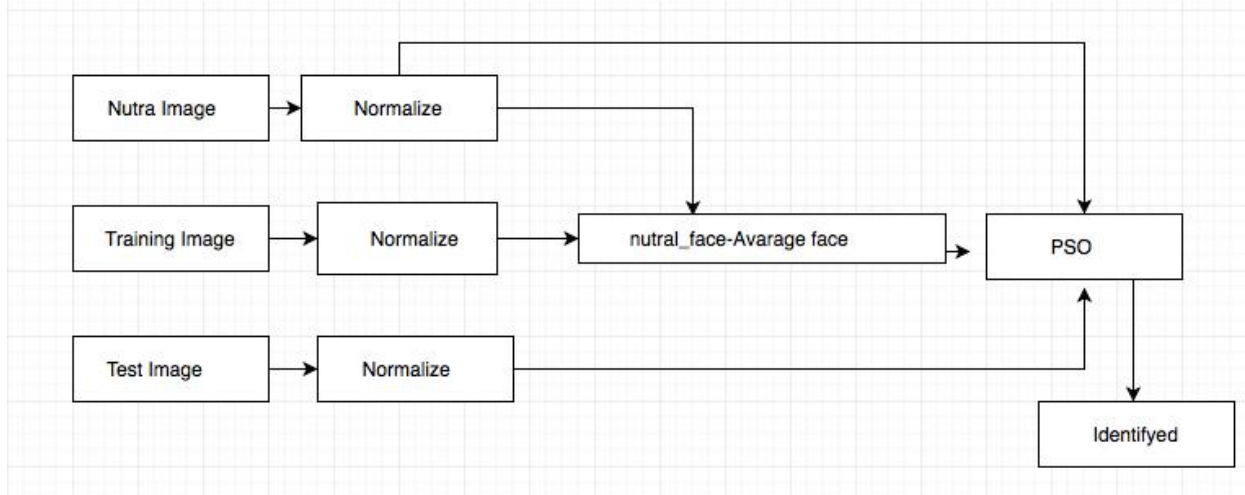

\section{Algorithm: OSPP using PSO}

In OSPP-PSO, Neutral image of each subjects are considered as Particles, and $V=[$ Neutral face $(i)-$ Average face $(i)$, are the rate of position change of particles'. Optimum solution is the minimum L2 distance of query image with particles (Neutral image). The Optimum solution can be achieved after a certain number of iterations in PSO.

Step1: input : Rate of Position Change of Each Particles $V_{i} \in I R^{m}$.Neutral Images $X_{1}^{i} \in I R^{m}$, $i=1,2,3 \ldots \ldots . K, K \quad$ isfor Subjects, and Query image $q \in I R^{m}$ and iteration $t=1$

Step2: initiate the neutral image as particles for each subjects $X=\left[X_{1}^{1}, X_{1}^{2} \ldots . . X_{1}^{k}\right]$

Step3: Position change of each particle in first iteration $V_{i, 1}=V_{r} \mathrm{r}$ is the random integer range between 1 to $k$, here $i$ represent the subject and 1 is for first iteration

Step4: Evaluate the L2 distance $D_{t}^{i}$ of $k$ subjects with quarry image $q$ where $i=1$ toK.initialize the L2 distance of $i$ th particle $D_{g, t}^{i}$ and minimum distance of whole Particle $D_{g, t}$

$$
\begin{gathered}
D_{t}^{i}=\left\|q-X_{t}^{i}\right\|_{2}, \quad i=1,2,3 \ldots k \ldots(3) \\
D_{g, t}=\text { inf } ; \\
\text { if } t=1, \text { then } D_{g, t}^{i}=D_{t}^{i} ;
\end{gathered}
$$

Step5: Update the best Point for Particle $i, P_{g, t}^{i}$ and update best point among all the particle point $P_{g, t}$ such as

$$
\begin{gathered}
\text { if } D_{t}^{i}<D_{g, t, t}^{i} \text { then } D_{g, t}^{i}=D_{t}^{i} \text { and } P_{g, t}^{i}=P_{t}^{i} . \\
D_{t}^{i}<D_{g, t} \text { then } D_{g, t}=D_{t}^{i} \text { and } P_{g, t}=P_{t}^{i} \ldots \ldots
\end{gathered}
$$

Step6: change the velocity and Position for particle using

$$
\begin{gathered}
V_{i, t+1}=w * V_{i, t}+C_{1} * \operatorname{rand}\left(\underset{()}{)} *\left(P_{g, t}^{i}-X_{t}^{i}\right)+C_{2} * \operatorname{rand}(\quad) *\left(P_{g, t}-X_{t}^{i}\right) \ldots .(1)\right. \\
X_{t+!}^{i}=X_{t}^{i}+V_{i, t+1} \ldots \ldots .(8)
\end{gathered}
$$

where $C_{1}, C_{2}$ are positive constant. and rand() is a random function.

Step7: $t=t+1$ and go to Step:5 after maximum number of iteration particale will get optimum fitness

Step8:Output: $\operatorname{Identity}(q)=\operatorname{Identity}\left(P_{g, t}\right)$

\section{Results \& Discussion}

The algorithm has been tested with the extended Yale face database B. The Yale face database B includes 39 subjects [YealB01 to YealB39] where each subject includes 64 pictures. Each sample picture is separated into two categories where 10 of the pictures are used for training purpose and the rest for testing purpose. yealBi_P00A+00E+00 was taken as neutral image for each subject. All samples were down sampled as $[24,21]$ and stacked row wise.In the experiment,number of iterations was set to 100 . The experiments were performed with 4 subjects and 10 test samples each. It was done using both the intra-class variety model and the proposed model with 10 test samples for each subject. Out of 40 attempts the intra-class variety model gave 36 accurate results which means that the model has $90 \%$ accuracy. In the proposed model out of 40 attempt 32 was found accurate which implies that out proposed model has $80 \%$ accuracy. 


\section{FIGURES AND TABLES}

\begin{tabular}{|c|c|c|c|c|c|}
\hline | & & & & A & B \\
\hline Subject & Sarpie tise for Training & Xutnel lmage & Sample tse for Thíring & Result & Kesult: \\
\hline \multirow{10}{*}{ yeleBO1 } & yleB01 P00A-005E -10 & \multirow{10}{*}{ yleBO1. $\mathrm{POOA}+00 \mathrm{E}+\infty$} & yaleBO1 POQA-035E +15 & $+v e$ & + ve \\
\hline & yaleB01.P00A-005E +10 & & raleBO1.POOA-0NoE +00 & twe & +ve \\
\hline & yaleBO1.POOA-010E-20 & & yaleBO1.POOA-070E-35 & $+n$ & +ve \\
\hline & yaleB01 $-900 \mathrm{~A} \cdot 010 \mathrm{E}+\infty$ & & yaleBOL $P 00 A-060 \mathrm{E}+20$ & $\cdot v$ & + ve \\
\hline & yleB01 P00A-015E+20 & & yaleBO1 P00A-060E-20 & tre & tve \\
\hline & yLeB01.P00A-020E-10 & & yaleBOL.POOA-050E +00 & $-v$ & twe \\
\hline & yLeB01.P0AA-C20E-60 & & yaleBOL.POOA-050E-40 & -ve & twe \\
\hline & yaleBO1 $P 00 A-020 \mathrm{E}+10$ & & yaleBO1 P00A-035E+65 & $+v e$ & tre \\
\hline & yebO1 POA $-025 E+\infty$ & & yaleBO1 POOA-035E +40 & twe & $-v e$ \\
\hline & yaleBOI.P00A-coss:-20 & & yaleBO1.POOA-035E+15 & $+\sqrt{+10}$ & +ve \\
\hline \multirow{10}{*}{ yakBO2 } & yaleBO2 P00A-005E-10 & \multirow{10}{*}{ yleBO2 $\mathrm{P} 00 \mathrm{~A}+000 \mathrm{E}+\infty$} & yaleBN2 P00A-035E +15 & $+v e$ & + ve \\
\hline & yale $B O 2 P 00 A-005 E+10$ & & yaleBN2 POOA-07OE- +00 & $+\mathrm{we}$ & $+v e$ \\
\hline & yeleBO2.P00,-010E-20 & & yaleBN2.POOA-0TOE-35 & twe & tre \\
\hline & yaleBO2.P00A-010E $+\infty$ & & yaleBO2.P00A-000E +20 & twe & $+\mathrm{ve}$ \\
\hline & yaleBO2 P00A-015E +20 & & yaleBC2 POOA-060E, 20 & $+\infty e$ & tve \\
\hline & yaleBO2.P00A-C20E-10 & & yaleBN2 P00A-050E-00 & twe & $+v e$ \\
\hline & yleBO2.P00A-020E- -10 & & yaleBO2.POCA-050E-40 & twe & +ve \\
\hline & yaleBO2.POAA-O20E+10 & & yaleBO2.POOA-035E +65 & $+v e$ & tre \\
\hline & yaleBO2 P00A-025E $+\infty$ & & yaleBO2 P0OA-105E +40 & $+v e$ & + we \\
\hline & yaleBO2 P00A-CosE-20 & & yaleBO2 P00A-035E +15 & the & tve \\
\hline \multirow{10}{*}{ yaleBcs } & yaleBO3.P00A-005E-10 & \multirow{10}{*}{ yaleB23.P0OA $+000 \mathrm{E}+\infty$} & yaleB03 P00A-035E +15 & $\cdot v$ & $-1 \mathrm{e}$ \\
\hline & yaleBC3 P00A-005E +10 & & yaleBC3 POOA-070E +00 & $\cdot v e$ & - - ve \\
\hline & yaleBC3 POOA-010E-20 & & yaleBC3.POOA-ONOE-35 & - ve & $-v e$ \\
\hline & yleBO3.P00A-010E +00 & & yaleB $03 . P 00 A-060 E+20$ & the & $+w$ \\
\hline & yaleBC3.P00A-015E +20 & & yaleBC3.P0OA-060E-20 & $+w$ & +ve \\
\hline & yaleBO3 $\mathrm{POOA}-020 \mathrm{E}-10$ & & yaleBC3 $P 00 A-050 E-00$ & $+\mathrm{we}$ & $+v e$ \\
\hline & yileB03 P00A-O20E- -10 & & yaleBC3 POOA-050E-40 & $+w$ & twe \\
\hline & yaleBC3.P00A-020E +10 & & yaleBC3.POOA-035E-65 & $\cdot 5$ & $+v e$ \\
\hline & yaleBC3.P00A-025E $+\infty$ & & yaleBC3.POCA-035E +40 & $\cdot v e$ & $+v e$ \\
\hline & yaleBO3 P00A-035E-20 & & yale BO3 P00A-035E +15 & twe & + ve \\
\hline \multirow{10}{*}{ yeleBO4 } & yaleBO4.P0A-005E-10 & \multirow{10}{*}{ yeleBQ4.P00A $+000 \mathrm{E}+\infty 0$} & yaleBO4.POOA-035E +15 & twe & $+v e$ \\
\hline & yaleBO4 P00A-005E +10 & & yaleBO4 POOA-0NOE +00 & $+n$ & tve \\
\hline & yaleBO4 POOA-010E-20 & & yaleBO4 POOA-OTOE-35 & $+n$ & tre \\
\hline & yaleBO4.P00A-010E+ $+\infty$ & & yaleBO4.P0OA-060E +20 & +we & tve \\
\hline & yaleBO4.P00A-015E+20 & & yaleBO4.POOA-060E-20 & twe & tve \\
\hline & yele $B 04$ POAA-020E-10 & & yaleBOA POOA-050E +00 & twe & +ve \\
\hline & yaleBO4 P00A-O20E-10 & & yaleBO4 POOA-050E-40 & ine & tve \\
\hline & yLeBOA.POOA-O20E+10 & & yaleBO4.POAS-0358+65 & Hese & (ivp) \\
\hline & yaleBOA.P00A-O25E $+\infty$ & & yaleBO4.POCA-035E +60 & $+v$ & the \\
\hline & yaleBO4.POOA-035E-20 & & yaleBO4.PUeA-035E+ 15 & 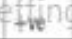 & -10 \\
\hline
\end{tabular}

Here Result (A) shows the the performance of the proposed Model and $\operatorname{Result}(\mathbf{B})$ shows the performance of intra-Class variation Model

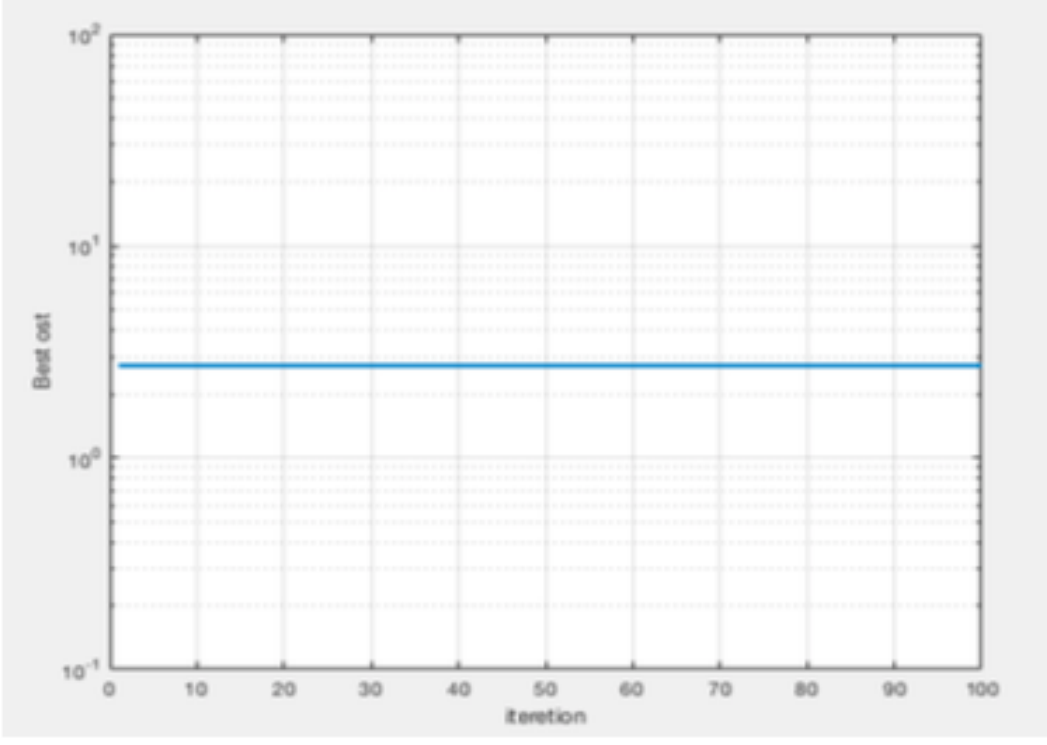

Fig: Performance Graph for PSO in OSPP 


\section{Conclusion}

A new OSPP face recognition algorithm is thus proposed. As its been already said that One sample per person face recognition (OSPP) is considered as a challenging problem in face recognition community and lack of samples leads to performance deterioration. Here face recognition is performed by application of the swarm optimization algorithms. To find the optimum point in terms of minimum L2 distance PSO is selected with the query image. Particle Swarm Optimization is used for optimally controlling the non-linear systems. It is notably easy to program and implement using logical and mathematical operations. PSO is one of the meta-heuristic algorithm that has been found to be robust in solving non-linear optimization problems. It is capable of generating high quality solutions with more stable convergence characteristics and shorter calculation time while compared to the others. PSO's performance remains robust even when applied to relatively high dimensions as typically found in control applications. The identity of the query image is equal to the identity of the basis image of the optimum combination. One sample face image of individual is been used for training the whole system which will both reduce labouring effort for the collection and also reduce cost for storing and processing.

\section{Acknowledgement}

I take this momentous opportunity to express our heartfull gratitude and regards to vulnerable and highly esteemed guide Mr. Priyaranjan Mishra. I am thankful to our colleagues whoprovided expertise that greatly assisted the research, although they may not agree with all of the interpretations provided in this paper. I also express out appreciation to my dearest friend for sharing their pearls of wisdom with us during the course. Nobody has been more important to me in the pursuit of this project than the members of my family. I would like to thank my parents, whose love and guidance are with me in whatever I pursue. They are the ultimate role models.

\section{References}

[1] J. Wu, Z.-H. Zhou, Face recognition with one training image per person, Pattern Recognition Lett. 23 (14) (2002) 1711-1719.

[2] J. Yang, D. Zhang, A.F. Frangi, J. Yang, Two-dimensional PCA: a new approach to appearance-based face representation and recognition, IEEE Trans. Pattern Anal. Mach. Intell. 26 (1) (2004) 131-137.

[3] B.S.Manjunath, R. Chellappa, C.V.D. Malsburg, A feature based approach to face recognition, in: Proceedings, IEEE Conference on Computer Vision and Pattern Recognition, vol. 1, 1992, pp. 373-378.

[4] M. Lades, J. Vorbruggen, J. Buhmann, J. Lange, Malsburg von der, R. Wurtz, Distortion invariant object recognition in the dyn amic link architecture, IEEE Trans. Comput. 42 (3) (1993) 300-311.

[5] L.Wiskott, R. Fellous, N. Kruger, C. von Malsburg, Face recognition by elastic bunch graph matching, IEEE Trans. Pattern Anal. Mach. Intell. 19 (7) (July 1997) 775-779.

[6] B.Kepenekci, F.B. Tek, G. BozdagiAkar, Occluded face recognition based on Gabor wavelets, ICIP 2002, September 2002, Rochester, NY, MP-P3.10.

[7] H.-S. Le, H. Li, Recognizing frontal face images using hidden Markov models with one training image per person, Proceedings of the 17th International Conference on Pattern Recognition (ICPR04), vol. 1, 2004, pp. 318-321.

[8] A.M. Martinez, Recognizing imprecisely localized, partially occluded, and ex- pression variant faces from a single sample per class, IEEE Trans. Pattern Anal. Mach. Intell. 25 (6) (2002) 748-763.

[9] S. Lawrence, C.L. Giles, A. Tsoi, A. Back, Face recognition: a convolutional neural-network approach, IEEE Trans. Neural Networks 8 (1) (1997) 98-113.

[10] X. Tan, S.C. Chen, Z.-H. Zhou, F. Zhang, Recognizing partially occluded, ex- pression variant faces from single training image per person with SOM and soft kNN ensemble, IEEE Trans. Neural Networks 16 (4) (2005) 875-886.

[11] YanZhang,HuaPeng,One sample per person face Recognition Using PSO,IET signal processing,pp 1-6,2015.

[12] James Kennedy, and Russel Eberhart (1995), Particle Swarm Optimizetion,IEEE 